\title{
Translation, Cross-cultural Adaptation, and Psychometric Properties of the Turkish Version of the Self-care Ability Scale for the Elderly
}

\author{
Mehmet Özkeskin ${ }^{1}$, Fatih Özden ${ }^{2}$, Sevnaz Şahin ${ }^{3}$ \\ ${ }^{1}$ Department of Physiotherapy and Rehabilitation, Faculty of Health Sciences, Ege University, Izmir, Turkey \\ ${ }^{2}$ Department of Elderly Care, Koycegiz Vocational School of Health Services, Mugla Sitki Kocman University, Mugla, Turkey \\ ${ }^{3}$ Division of Geriatrics, Department of Internal Medicine, Faculty of Medicine, Ege University, Izmir, Turkey
}

Corresponding Author:

Fatih Özden, PT, MSc

Department of Elderly Care, Koycegiz

Vocational School of Health Services,

Mugla Sitki Kocman University, 48800,

Koycegiz, Mugla, Turkey

E-mail: fatihozden@mu.edu.tr

ORCID:

https://orcid.org/0000-0001-6593-3758

Received: April 29, 2021

Revised: June 8, 2021

Accepted: June 9, 2021
Purpose: The Self-care Ability Scale for the Elderly (SASE) is a frequently used scale that was created in Sweden according to Orem's self-care deficit theory and later modified according to Pörn's theory of environment and purpose. This study translated and cross-culturally adapted the Turkish version of the SASE (T-SASE) and demonstrated its psychometric properties, including its reliability and construct validity. Methods: This study recruited a total of 122 participants and applied international guidelines for the translation and adaptation of the scale. The test-retest reliability of the SASE was calculated at 1-week intervals. Internal consistency was analyzed using Cronbach's alpha. For construct validity, we compared the T-SASE to the Nottingham Extended Activities of Daily Living Scale (NEADLS) and the Exercise of Self-Care Agency Scale (ESCAS). Results: The mean age of the participants was $68.6 \pm 5.7$ years. The test-retest reliability of the T-SASE was excellent (intraclass correlation coefficient $=0.914 ; 95 \%$ confidence interval, $0.81-$ 0.95). The internal consistency of the total T-SASE score was also excellent (Cronbach's $\alpha=0.912$ ), as were the Cronbach's alpha scores for the individual items, ranging from 0.901 to 0.915 . The items were also highly consistent with each other (Cronbach's $\alpha>0.80$ ). The construct validity of the T-SASE was acceptable $(0.35<r<0.50)$ in a convergent manner. The T-SASE was also strongly correlated with the NEADLS and ESCAS $\left(r_{1}=0.405, r_{2}=0.437, p<0.01\right)$. Conclusions: The T-SASE was a reliable and valid tool for assessing the self-care ability of community-dwelling older adults. Owing to its multidimensional structure, the T-SASE is an essential tool for evaluating self-care in older adults.

Key Words: Psychometrics, Self-Care, Aptitude, Surveys and Questionnaires

\section{INTRODUCTION}

The United Nations estimated that the number of people aged 60 and over will exceed 2 billion in 30 years. Therefore, evaluating self-care in this older population is of particular importance. ${ }^{1)}$ The aging process includes a decrease in the functional level as well as physiological deficiencies. In the process of aging, some restrictions occur in daily living activities. ${ }^{2,3)}$ Self-care ability is an essential determinant of the maintenance of daily life activities in older adults. ${ }^{4)}$ Self-care is defined as personal care initiated by individuals on their behalf to maintain their health and well-being, while selfcare ability is defined as the ability to perform self-care or self-management activities. ${ }^{5)}$ Self-care in older adults encompasses both self-care ability and process. Self-care ability includes self-care activities to identify individual needs, evaluate internal and external resources, and provide functional independence. ${ }^{6}$ ) Self-care ability is performed under certain environmental conditions and using various devices for a specific purpose. Personal care activities (e.g., nutrition, dressing, and body cleaning), home cleaning, transportation, and shopping are generally performed by a device. ${ }^{4)}$ Person- 
al care activities can be affected by many conditions, such as health, good habits, self-esteem, and self-care ability. ${ }^{7)}$ However, advanced age negatively affects self-care, ${ }^{8)}$ while the ability to perform daily life activities and level of education positively affect self-care. ${ }^{9,10)} \mathrm{A}$ person's self-care ability may differ in different developmental stages and in acute or chronic health conditions. ${ }^{7)}$ Self-care ability is an essential source of health in older adults, especially those living in their own homes, ${ }^{6}$ and also enables older people with self-care ability to manage their existing diseases and develop their behaviors. ${ }^{11)}$ Increasing self-care ability may support disease prevention and healing and improving individuals' quality of life. ${ }^{12,13)}$

Health professionals should be encouraged to improve the selfcare ability of older adults, which requires evaluation of this ability of older adults at individual and social risk and the development of treatment plans. ${ }^{14,15)}$ Various tools for assessing self-care have been described in the literature. Tools used for older adults ${ }^{16)}$ include the Self-care of Home-Dwelling Elderly Instrument, Lorensen's Self-Care Capability Scale, and the Self-care Ability Scale for the Elderly (SASE) ${ }^{17-19)}$ The Self-care of Home-Dwelling Elderly Instrument and Lorensen's Self-Care Capability Scale contain 82 and 56 questions, respectively, which impose a burden to complete. ${ }^{17,18)}$ In contrast, the SASE has relatively fewer questions and is widely used for clinical evaluation. ${ }^{20)}$

The SASE was created in Sweden according to the Self-Care Deficit Theory and later modified according to Pörn's theory of environment and purpose. ${ }^{4,14)}$ The SASE originally contained 53 items, which was reduced to 17 . The Cronbach's alpha coefficients ranged from 0.44 to 0.88 for the five factors of the SASE and between 0.70 and 0.80 for three factors. ${ }^{20)}$ In their systematic review, Matarese et al. ${ }^{16)}$ found strong evidence of SASE content validity and moderate evidence of construct validity and hypothesis testing. However, they cited contradictory evidence regarding the internal consistency of the SASE. The items in the SASE measure the ability of older individuals to perform daily life activities, their health and life satisfaction experiences, and their purpose in performing some daily life activities. ${ }^{20)}$ The validity and reliability of the Swedish version of the SASE have been reported. ${ }^{4,20)}$ The SASE was later translated into Chinese, ${ }^{21)}$ Norwegian, ${ }^{22)}$ Italian, ${ }^{23)}$ and $\operatorname{Iranian}^{24)}$ languages, and its psychometric properties were examined. However, to our knowledge, the validity and reliability of a Turkish version have not been established. The psychometric study of the Turkish version of SASE can be used in clinical studies to evaluate the self-care skills of Turkish-speaking healthy older individuals. In addition, it would be a preferable scale for evaluating geriatric rehabilitation programs in clinical training provided to older adults.

This study aimed to translate the SASE into Turkish, adapt it culturally, and test its reliability and validity in healthy older individuals.

\section{MATERIALS AND METHODS}

\section{Translation and Adaptation Process}

To examine the psychometric properties of the SASE, permission to use the scale was obtained from Ulrika Söderhamn who developed it. ${ }^{20)}$ The scale's cultural adaptation was carried out according to internationally accepted translation procedures. ${ }^{25)}$ In the first stage, the advanced translation stage, the original Swedish version of the SASE was translated into Turkish independently by two professional bilingual translators whose native language was Turkish and who were also proficient in Swedish. Expert committee members (two academician physiotherapists) identified and recorded problems related to culture and linguistics. In the second stage, the same expert committee discussed the correction notes for these translations. At this stage, the third item was modified. The committee added the term "dentures, if any" to the end of the sentence with parenthesis. Additionally, the word "house-keeping" mentioned in the 4th and 13th items is used more frequently in European countries, while "house cleaning" is more commonly used in Turkish. Therefore, we used the term "ev temizliği" ("house cleaning") instead of "house-keeping." In the third stage, the SASE was translated into Swedish independently by two translators whose mother tongue was Swedish. The original Swedish SASE was compared to the back-translated version. In the fourth stage, conceptual and linguistic issues were discussed. In the fifth stage, a draft version was produced for use in pilot testing. In the last stage, a pilot study was conducted to determine whether the Turkish version of the SASE provided suitable understandability. A pre-test was conducted on a 5-point Likert-type scale with 30 older adults speaking Turkish. The understandability was excellent in this pilot study. Therefore, no additional changes were made. Finally, the Turkish version of SASE (T-SASE) was created.

\section{Ethical Statement}

The permission for the translation for the Turkish version of the Self-care Ability Scale was acquired from the developer of the original questionnaire. The study was carried out in accordance with the ethical principles and the Helsinki Declaration. Informed consent of the patients was obtained. The study protocol was approved by the ethics committee of Ege University (No. 21-3T/22).

\section{Sample Size Estimation}

Terwee et al. ${ }^{26)}$ recommended at least 100 patients for the assessment of the internal consistency of health-related patient-reported 
outcome measures (PROMs). In addition, the required sample size to analyze the T-SASE reproducibility was calculated using the $\mathrm{G}^{*}$ Power 3.1 software with an effect size of 0.50 , a probability of error of 0.05 , and a power of $0.80 .^{27)}$ The effect size for test-retest reliability analysis (i.e., reproducibility) was determined using Cohen's d coefficient (0.50), which indicates a medium-sized standardized difference between test and retest. ${ }^{28)}$ In conclusion, at least 21 patients were required for the reliability analysis. Thus, 30 patients repeated the T-SASE 1 week after the initial assessment.

\section{Study Design}

This prospective cross-sectional study conducted by the Department of Geriatrics at Ege University included healthy older individuals. The following older individuals were included in this study: (1) older individuals aged 65 years and older and (2) those who could read, understand, and speak Turkish. Older individuals (1) with acute or chronic diseases that could affect their self-care, (2) those who were bedridden, or (3) those who did not agree to participate in the study were excluded.

The sociodemographic and physical characteristics of the older individuals participating in the study were collected. The Turkish versions of the Nottingham Extended Activities of Daily Living Scale (NEADLS) ${ }^{29)}$ and the Exercise of Self-Care Agency Scale $(\text { ESCAS })^{30)}$ were performed as a parallel form to assess SASE's structural validity. In the first evaluation, 122 older individuals answered all the items of the T-SASE, NEADLS, and ESCAS in the given order. The retest evaluation of the T-SASE was conducted 1 week later with 30 older individuals.

\section{SASE}

The SASE is a self-report tool developed by Söderhamn to measure self-care ability in older individuals. The SASE consists of 17 items related to daily life activities, well-being, mastery, willpower, determination, loneliness, and dressing. Each item's score ranges from 1 to 5 ( 1 "completely disagree" to 5 "completely agree"). Three points were considered neutral scores. Items containing negative statements are summed by reversing the score. The total score ranged from 17 to 85 , with higher scores indicating increased self-care ability. ${ }^{4)}$

\section{NEADLS}

The NEADLS was developed to evaluate daily living activities in rehabilitation centers in England. ${ }^{31)}$ The NEADLS consists of four subsections containing questions about mobility (six items), kitchen (five items), housework (five items), and leisure activities (six items). The answers to all questions are evaluated as follows: 0 points, never done; 1 point, can be done with help; 2 points, can be done by force; and 3 points, can be done quickly without assistance. A final score ranging from 0 to 66 points was obtained by summing the scores from each subsection. This scale can also be conducted through clinician interview or by mail. ${ }^{29)}$

\section{ESCAS}

The scale, consisting of 35 items, was intended to determine individuals' self-care abilities. The scale is evaluated using a 5-point Likert scale $(0=$ does not describe me at all, $1=$ does not describe me much, 2 = I have no idea, 3 = describes me a little, $4=$ describes me very well). Items \#3, \#6, \#9, \#13, \#19, \#22, \#26, and \#31 on the scale have negative meanings and are evaluated as negative. The highest possible score on the ESCAS is $172 .^{30)}$

\section{Statistical Analysis}

All data collected from the research were analyzed using IBM SPSS for Windows version 25.0 (IBM Corp., Armonk, NY, USA). Means and standard deviations were calculated for quantitative data and percentages for qualitative data. Shapiro-Wilk tests were used to test the data for a homogeneous distribution. The minimum and maximum T-SASE scores were also analyzed for possible floor or ceiling effects. In addition, 95\% confidence intervals (CIs) were used to calculate the correlation coefficients. Internal consistency was analyzed by calculating the Cronbach's alpha of the total score and all items of the T-SASE, with alpha values above 0.6 and 0.8 representing good and excellent internal consistency, respectively. ${ }^{32)}$ For test-retest reliability, the intraclass correlation coefficient (ICC) was calculated for the total T-SASE score, with an ICC value over 0.8 indicating perfect reproducibility. ${ }^{33)}$ The construct validity of the T-SASE was analyzed by correlation with the NEADLS and ESCAS based on Pearson correlation coefficient $(r)$. The coefficient was considered high for values above 0.5 , moderate for values between 0.5 and 0.35 , and low for values below 0.35 . $^{34)}$

\section{RESULTS}

The mean age of the older adults was $68.6 \pm 5.7$ years. This study included a total of 84 women (68.9\%) and 38 men (31.1\%). A larger number of the participants had a bachelor's or higher degree. More than half of the participants were married and lived with their families (59\% and 64.8\%, respectively). Most of the older adults were able to walk independently without an assistive device (95.9\%). Similarly, most participants did not have a fall history (75.4\%). The participant characteristics are presented in Table 1. The absolute values of the PROM measurements of older adults are presented in Table 2 . 
Table 1. Patients' characteristics $(n=122)$

\begin{tabular}{lc}
\hline & Value \\
\hline Age $(\mathrm{y})$ & $68.6 \pm 5.7$ \\
$\mathrm{BMI}\left(\mathrm{kg} / \mathrm{m}^{2}\right)$ & $26.7 \pm 4.0$ \\
Sex & \\
Female & $84(68.9)$ \\
Male & $38(31.1)$ \\
Education & \\
Primary school & $29(23.8)$ \\
Middle school & $7(5.7)$ \\
High school & $13(10.7)$ \\
University or higher degree & $73(59.8)$ \\
Marital status & \\
Married & $72(59.0)$ \\
Single & $50(41.0)$ \\
Residence & \\
Family & $79(64.8)$ \\
Alone & $37(30.3)$ \\
Other & $6(4.9)$ \\
Chronic diseases & \\
Yes & $69(56.6)$ \\
No & $53(43.4)$ \\
\hline
\end{tabular}

Values are presented as mean \pm standard deviation or number (\%).

BMI, body mass index.

The test-retest reliability of the T-SASE was excellent (ICC $=0.914 ; 95 \% \mathrm{CI}, 0.81-0.95$ ). In addition, the internal consistency of the total T-SASE score was excellent (Cronbach's $\alpha=0.912$ ). The Cronbach's alpha scores for the individual items were also excellent, ranging from 0.901 to 0.915 and the items were highly consistent with each other (Cronbach's $\alpha>0.80$ ) (Table 3). The construct validity of the T-SASE was acceptable $(0.35<\mathrm{r}<0.50)$ in a convergent manner. The T-SASE was strongly correlated with the NEADLS and ESCAS $\left(r_{1}=0.405, r_{2}=0.437, p<0.01\right)$ (Table 4$)$.

\section{DISCUSSION}

The present study aimed to develop and cross-culturally adapt a Turkish version of the SASE. We also aimed to demonstrate the reliability and validity of the T-SASE. Considering the importance of standardized and adapted PROMs in geriatric occupational therapy, the T-SASE is an essential tool for evaluating the self-care ability in older adults in performing activities of daily living and to identify individual care issues. ${ }^{14,20)}$ Our results showed that the T-SASE was a reliable and valid scale for use in community-dwelling older adults. The internal consistency and test-retest reliability of the T-SASE were high, and the validity was acceptable in the construct validity analysis in terms of convergent substance.
Table 2. Absolute values of the patient-reported outcome measures $(n=122)$

\begin{tabular}{lc}
\hline & Value \\
\hline T-SASE & $71.43 \pm 8.49(29-80)$ \\
NEADLS & $61.06 \pm 6.45(32-66)$ \\
ESCAS & $122.09 \pm 14.22(69-140)$ \\
\hline
\end{tabular}

Values are presented as mean \pm standard deviation (range).

T-SASE, Turkish version of the Self-care Ability Scale; NEADLS, Nottingham Extended Activities of Daily Living Scale; ESCAS, Exercise of Self-Care Agency Scale.

Table 3. Test-retest reliability and internal consistency of the T-SASE $(n=30)$

\begin{tabular}{lccc}
\hline Item\# & Test & Retest & Cronbach's $\alpha$ \\
\hline 1 & $4.33 \pm 1.04$ & $4.16 \pm 1.11$ & 0.907 \\
2 & $4.71 \pm 0.70$ & $4.83 \pm 0.37$ & 0.901 \\
3 & $4.75 \pm 0.69$ & $4.80 \pm 0.40$ & 0.903 \\
4 & $4.63 \pm 0.81$ & $4.36 \pm 0.80$ & 0.903 \\
5 & $4.71 \pm 0.68$ & $4.66 \pm 0.47$ & 0.901 \\
6 & $4.31 \pm 0.61$ & $3.40 \pm 1.21$ & 0.913 \\
7 & $4.20 \pm 0.97$ & $4.06 \pm 0.78$ & 0.910 \\
8 & $4.45 \pm 0.83$ & $4.40 \pm 0.49$ & 0.905 \\
9 & $4.36 \pm 0.86$ & $4.26 \pm 0.58$ & 0.903 \\
10 & $4.24 \pm 0.91$ & $4.36 \pm 0.71$ & 0.905 \\
11 & $4.54 \pm 0.80$ & $4.26 \pm 0.94$ & 0.903 \\
12 & $4.22 \pm 1.12$ & $4.36 \pm 0.71$ & 0.913 \\
13 & $4.39 \pm 0.96$ & $4.23 \pm 1.04$ & 0.904 \\
14 & $4.20 \pm 0.60$ & $3.20 \pm 1.06$ & 0.915 \\
15 & $4.39 \pm 0.74$ & $4.36 \pm 0.66$ & 0.905 \\
16 & $4.40 \pm 0.63$ & $4.16 \pm 0.74$ & 0.911 \\
17 & $4.74 \pm 0.76$ & $4.83 \pm 0.37$ & 0.906 \\
T-SASE & $71.43 \pm 8.49$ & $72.53 \pm 5.07$ & 0.912 \\
\hline
\end{tabular}

Values are presented as mean \pm standard deviation.

T-SASE, Turkish version of the Self-care Ability Scale.

Table 4. Correlations between the NEADLS and ESCAS with T-SASE $(n=122)$

\begin{tabular}{lcc}
\hline & $\mathrm{r}$ & $\mathrm{p}$-value \\
\hline T-SASE - NEADLS & 0.405 & $<0.01$ \\
T-SASE - ESCAS & 0.437 & $<0.01$ \\
\hline
\end{tabular}

r, Pearson correlation coefficient; T-SASE, Turkish version of the Self-care Ability Scale; NEADLS, Nottingham Extended Activities of Daily Living Scale; ESCAS, Exercise of Self-Care Agency Scale.

In terms of self-care, the independence of older adults is essential and is primarily related to their quality and adeptness of life. Developed by considering Pörn's health and adaptation theory, the SASE is widely used to measure the self-care capacity of older adults in a standardized, practical, and accurate manner. ${ }^{4,1420)}$ However, it may not be sufficient to express only functional ability in evaluating 
self-care ability, and independence in daily living activities is adequate. This assessment must include both cognitive and emotional components. Unlike the current tools to assess activities of daily living and self-care, the T-SASE provides a more specific self-care assessment, including these parameters, especially in older adults, who often have cognitive and depressive problems. ${ }^{20}$ )

The original Swedish version of the SASE has been translated to Chinese, Italian, Norwegian, and Persian. ${ }^{20-24)}$ The culturally adapted versions are reliable, valid, and frequently used tools to assess self-care ability in older adults. Our study translated and adapted the SASE into Turkish according to internationally accepted guidelines. ${ }^{25)}$ During this process, only one modification was carried out for item \#3, which assesses oral hygiene, in which the term "varsa diş protezi" is given in parentheses. We put the word "dentures, if any" before this expression in Turkish to preserve the grammatical structure of the questionnaire.

The reliability of the T-SASE was assessed by analyzing its internal consistency and test-retest reliability. The internal consistencies of the total score and the items of T-SASE were excellent $(>0.80)$. The SASE comprises items related to the functional, cognitive, and emotional components of self-care ability. This versatile tool's capacity to evaluate self-care activities holistically and consistently is essential for its reliability. ${ }^{4)}$ Thus, we analyzed the internal consistency of the total score and the items' independent alpha scores and found that the T-SASE evaluated older individuals consistently. The items of the scale were also consistent with each other to assess self-care in a three-dimensional manner. The original Swedish version showed Cronbach's alpha values of 0.68 and 0.88 in older patients and community-dwelling older adults, respectively. ${ }^{20)}$ The Cronbach's alpha value for the Italian version ranged from 0.72 to $0.90,{ }^{23)}$ while the values for the Chinese, Norwegian, and Persian versions were $0.89,0.85$, and 0.73 , respectively. ${ }^{21,22,24)}$ The results demonstrated the acceptable to high consistency of the SASE in all cultures, including Turkey. Thus, this tool provides clinicians and researchers consistent assessment of the self-care ability of patients and older individuals.

The generally accepted retest time intervals reported in the literature range between 2 days and 2 weeks. ${ }^{26)}$ The test-retest reliability of the T-SASE total score was excellent $(>0.80)$; in other words, the T-SASE showed acceptable reproducibility. The T-SASE showed accurate results for measurements performed at different times. T-SASE provided the participants' actual score without bias and evaluation error. ${ }^{33)}$ The Chinese and the Italian versions showed ICC values of 0.99 and 0.92 , respectively, ${ }^{21)}$ while those of the Persian version ranged from 0.85 to $0.97 .^{24)}$ The original development study did not calculate ICC values. ${ }^{20)}$ These findings demonstrate the reliability of the SASE in terms of reproduc- ibility. The results of the Turkish version are similar to those of the other versions.

Construct validity is another essential component for revealing PROM psychometrics. Factor analysis or convergent-divergent validity by comparing to other standard tools are used for scale validation. ${ }^{34)}$ The present study focused on convergent validity, considering the structure of the SASE. Because similar tools that evaluate daily living activities focus only on functionality, we compared the SASE to the NEADLS. The primary goal was to evaluate the appropriateness of the self-care activities by multidimensional evaluation by comparing the T-SASE to a one-dimensional tool (NEADLS) ${ }^{29)}$ In addition, we examined the ability of the questioning self-care agency of SASE by comparing its concordance to a gold standard questionnaire (ESCAS). ${ }^{30)}$ The NEADLS does not comprehensively examine the health and life satisfaction of older individuals in terms of self-care. ${ }^{29)}$ The ESCAS, on the other hand, has a long structure, containing 35 items. ${ }^{30)}$ Therefore, the T-SASE may provide advantages over both questionnaires. Our validity results showed that the T-SASE was strongly correlated with the NEADLS and ESCAS $\left(r_{1}=0.405, r_{2}=0.437, p<0.01\right)$. Based on the commonly applied reference cut-off values for construct validity $(0.35<\mathrm{r}<0.50)$, the T-SASE showed acceptable validity. ${ }^{34)}$ None of the version studies and the original validation study reported the convergent validity with comparisons to other questionnaires. The Italian version compared the SASE to the Katz Index of Independence in Activities of Daily Living and the Lawton Instrumental Activities of Daily Living, in which the correlation coefficients ranged from 0.11 to 0.63 . $^{23)}$ Instead of the Katz and Lawton questionnaires, we compared the T-SASE to the NEADLS, the items of which were more consistent with those of the SASE. Both the Italian and Turkish versions of the SASE also showed acceptable validity.

This study had several limitations, which could inform the design of future SASE validation studies. First, confirmatory and explanatory factor analysis could be used to comprehensibly express the construct structure in assessing the SASE validity. However, as other versions adequately provided these analyses, ${ }^{21,24)}$ we focused more on convergent validity. Second, while this study recruited community-dwelling older adults, additional studies are needed to evaluate clinical populations and hospitalized older patients to demonstrate the psychometrics of SASE in detail. Third, the present and other versions have not evaluated the responsiveness of the SASE. Determining the relationships between clinical and other endpoints, as well as variations in SASE scores over time, will be essential. Finally, retest evaluations should be conducted at least 2 weeks later to avoid potential practice effects.

In conclusion, the results of this study showed that the T-SASE 
was a valid and reliable tool for assessing the self-care ability of community-dwelling older adults. Owing to its multidimensional structure (functionality, cognitive, and emotional), the SASE is an essential tool for geriatric rehabilitation specialists to evaluate daily living activities and daily self-care issues in older adults.

\section{ACKNOWLEDGMENTS}

\section{CONFLICT OF INTEREST}

The researchers claim no conflicts of interest.

\section{FUNDING}

None.

\section{AUTHOR CONTRIBUTION}

Conceptualization, FO, MO, SS; Data curation, MO, SS; Investigation, FO, MO, SS; Methodology, FO, MO; Project administration, FO, MO, SS; Supervision, FO, MO, SS; Writing-original draft, FO, MO, SS; Writing-review \& editing, FO, MO, SS.

\section{REFERENCES}

1. United Nations. World population prospects: key findings and advance tables (2015 revision). New York, NY: United Nations Publications; 2016.

2. Isik EI, Yilmaz S, Uysal I, Basar S. Adaptation of the Lawton instrumental activities of daily living scale to Turkish: validity and reliability study. Ann Geriatr Med Res 2020;24:35-40.

3. Bryant LL, Corbett KK, Kutner JS. In their own words: a model of healthy aging. Soc Sci Med 2001;53:927-41.

4. Suderhamn O, Ek AC, Purn I. The self-care ability scale for the elderly. Scand J Occup Ther 1996;3:69-78.

5. Dale B, Soderhamn U, Soderhamn O. Self-care ability among home-dwelling older people in rural areas in southern Norway. Scand J Caring Sci 2012;26:113-22.

6. Hoy B, Wagner L, Hall EO. Self-care as a health resource of elders: an integrative review of the concept. Scand J Caring Sci 2007;21:456-66.

7. Orem DE, Taylor SG, Repenning KM. Nursing concepts of practice. St. Louis, MO: Mosby; 2001.

8. Doran D. Nursing outcomes: the state of the science. Sudbury, MA: Jones \& Bartlett Learning; 2011.

9. Fagerstrom C, Holst G, Hallberg IR. Feeling hindered by health problems and functional capacity at 60 years and above. Arch Gerontol Geriatr 2007;44:181-201.

10. Newsom JT, Kaplan MS, Huguet N, McFarland BH. Health behaviors in a representative sample of older Canadians: prevalenc- es, reported change, motivation to change, and perceived barriers. Gerontologist 2004;44:193-205.

11. Sousa VD, Zauszniewski JA, Bergquist-Beringer S, Musil CM, Neese JB, Jaber AF. Reliability, validity and factor structure of the Appraisal of Self-Care Agency Scale-Revised (ASAS-R). J Eval Clin Pract 2010;16:1031-40.

12. Heidarzadeh M, Atashpeikar S, Jalilazar T. Relationship between quality of life and self-care ability in patients receiving hemodialysis. Iran J Nurs Midwifery Res 2010;15:71-6.

13. Lee BA, Leiby BE, Marino RJ. Neurological and functional recovery after thoracic spinal cord injury. J Spinal Cord Med 2016;39:67-76.

14. Soderhamn O, Lindencrona C, Ek A. Ability for self-care among home dwelling elderly people in a health district in Sweden. Int J Nurs Stud 2000;37:361-8.

15. Azadbakht M, Garmaroodi G, Taheri Tanjani P, Sahaf R, Shojaeizade D, Gheisvandi E. Health promoting self-care behaviors and its related factors in elderly: application of health belief model. J Educ Community Health 2014;1:20-9.

16. Matarese M, Lommi M, De Marinis MG. Systematic review of measurement properties of self-reported instruments for evaluating self-care in adults. J Adv Nurs 2017;73:1272-87.

17. Lorensen M. Ældre og egenomsorg [Older people and selfcare]. Kobenhavn, Denmark: Munksgaard; 1986.

18. Rasanen P, Backman K, Kyngas H. Development of an instrument to test the middle-range theory for the self-care of home-dwelling elderly. Scand J Caring Sci 2007;21:397-405.

19. Porn I. Health and adaptedness. Theor Med 1993;14:295-303.

20. Soderhamn O, Lindencrona C, Ek AC. Validity of two self-care instruments for the elderly. Scand J Occup Ther 1996;3:172-9.

21. Gao H, Soderhamn U, Cliffordson C, Guo L, Guo Q, Liu K. Reliability and validity of the Chinese version of the Self-care Ability Scale for the Elderly.J Clin Nurs 2017;26:4489-97.

22. Tomstad ST, Soderhamn U, Espnes GA, Soderhamn O. Testing two self-care-related instruments among older home-dwelling people in Norway. Int J Older People Nurs 2013;8:189-98.

23. Raffaele B, Biagioli V, Cirillo L, De Marinis MG, Matarese M. Cross-validation of the Self-care Ability Scale for Elderly (SASE) in a sample of Italian older adults. Scand J Caring Sci 2018;32:1398-1408.

24. Amir-Behghadami M, Tabrizi JS, Saadati M, Gholizadeh M. Psychometric properties of the Iranian version of self-care ability scale for the elderly. BMC Geriatr 2020;20:364.

25. Guillemin F, Bombardier C, Beaton D. Cross-cultural adaptation of health-related quality of life measures: literature review and proposed guidelines. J Clin Epidemiol 1993;46:1417-32.

26. Terwee CB, Bot SD, de Boer MR, van der Windt DA, Knol DL, 
Dekker J, et al. Quality criteria were proposed for measurement properties of health status questionnaires. J Clin Epidemiol 2007;60:34-42.

27. Faul F, Erdfelder E, Lang AG, Buchner A. G*Power 3: a flexible statistical power analysis program for the social, behavioral, and biomedical sciences. Behav Res Methods 2007;39:175-91.

28. Gignac GE, Szodorai ET. Effect size guidelines for individual differences researchers. Pers Individ Dif 2016;102:74-8.

29. Sahin F, Yilmaz F, Ozmaden A, Kotevoglu N, Sahin T, Kuran B. Reliability and validity of the Turkish version of the Nottingham Extended Activities of Daily Living Scale. Aging Clin Exp Res 2008;20:400-5.

30. Nahcivan NO. A Turkish language equivalence of the Exercise of Self-Care Agency Scale. West J Nurs Res 2004;26:813-24.
31. Turner-Stokes L, Turner-Stokes T. The use of standardized outcome measures in rehabilitation centres in the UK. Clin Rehabil 1997;11:306-13.

32. Schermelleh-Engel K, Moosbrugger H, Muller H. Evaluating the fit of structural equation models: tests of significance and descriptive goodness-of-fit measures. Methods Psychol Res Online 2003;8:23-74.

33. Baumgartner TA, Chung $\mathrm{H}$. Confidence limits for intraclass reliability coefficients. Meas Phys Educ Exerc Sci 2001;5:179-88.

34. Juniper EF. How to develop and validate a new health-related quality of life instrument. In: Spilker B, editors. Quality of life and pharmacoeconomics in clinical trials. Philadelphia, PA: Lippincott Williams \& Wilkins; 1996. p. 49-56. 